\title{
ANÁLISE DE CORRELAÇÕES ENTRE INDICADORES ECONÔMICOS: PIB, CESTA BÁSICA E IDH
}

\author{
Cleiton Landi de Oliveira, FATEC-Osasco, Brasil. \\ cleiton.oliveira8@fatec.sp.gov.br \\ Fábio Stuchi, FATEC-Osasco, Brasil \\ fabio.stuchi@fatec.sp.gov.br \\ Fernando de Almeida Santos, FATEC-Osasco e PUC-SP, Brasil \\ fernando@fernandoasantos.com.br
}

\section{RESUMO}

A pesquisa tem o objetivo de verificar a correlação, conforme a teoria do matemático Karl Pearson (1857-1936), entre as variáveis: Produto Interno Bruto (PIB), a Cesta básica e o Índice de desenvolvimento Humano (IDH). A pesquisa abordou o período de 2007 a 2.018, com variáveis anuais foi utilizada a teoria de Pearson. Ao final observa-se forte correlação entre o PIB e a Cesta Básica, mas não ocorre o mesmo entre as outras variáveis. Tal observação pode contribuir com a elaboração de políticas públicas, demonstrando que o aumento da produção nem sempre resulta em maior distribuição de renda e evolução da sociedade.

Palavras-Chave: PIB; indicadores; correlação.

Data de recebimento: 14/02/2021

Data do aceite de publicação: $15 / 04 / 2021$

Data da publicação: 30/04/2021 
ANÁLISE DE CORRELAÇÕES ENTRE INDICADORES ECONÔMICOS: PIB, CESTA BÁSICA E IDH

\title{
ANALYSIS OF CORRELATIONS BETWEEN ECONOMIC INDICATORS: GDP, BASIC BASKET AND HDI
}

\author{
Cleiton Landi de Oliveira, FATEC-Osasco, Brasil. \\ cleiton.oliveira8@fatec.sp.gov.br \\ Fábio Stuchi, FATEC-Osasco, Brasil \\ fabio.stuchi@fatec.sp.gov.br \\ Fernando de Almeida Santos, FATEC-Osasco e PUC-SP, Brasil \\ fernando@fernandoasantos.com.br
}

\begin{abstract}
The research aims to verify the correlation, according to the theory of the mathematician Karl Pearson (1857-1936), between the variables: Gross Domestic Product (GDP), the Basic Basket and the Human Development Index (HDI). The research covered the period from 2007 to 2,018, with annual variables Pearson's theory was used. In the end, there is a strong correlation between GDP and the Cesta Básica, however, the same not resulted among the other variables. Such observation can contribute to the elaboration of public policies, demonstrating that the increase in production does not always result in a greater distribution of income and evolution of society.
\end{abstract}

Keywords: GDP; Indicators; Correlation.

\section{INTRODUÇÃ̃}

Os indicadores podem auxiliar nas medições dos resultados de performances de sistemas econômicos, atualmente no Brasil usam-se o PIB, a Cesta básica e o IDH para apurações estatísticas da economia. A cesta básica nacional, elaborou-se no perfil de consumo interno, porém os outros dois indicadores, poderão serem comuns como ferramenta de controle em outras economias internacionais.

Os resultados apurados pelos indicadores auxiliam nos diagnósticos de cenários para as tomadas de decisões, sejam por governantes, empresários, investidores ou quem necessite da resposta de desempenho apontada pela mensuração do indicador apurado. Os papéis de facilitadores dos indicadores, talvez, ajudem nas tomadas de decisões mais assertivas; quando houver necessidade de traçar planos, estratégias ou estudos de investimentos.

Entre os indicadores do cenário brasileiro, escolhem-se para aplicações comparativas com base na teoria de correlação do matemático Karl Pearson (1857-1936). 
ANÁLISE DE CORRELAÇÕES ENTRE INDICADORES ECONÔMICOS: PIB, CESTA BÁSICA E IDH

O PIB para medir o desempenho econômico, por meio da produção nacional, a cesta básica para determinar os custos dos alimentos básicos consumidos por um indivíduo adulto e o IDH para indicar o desenvolvimento humano no país.

Entretanto, importa-se realizar as correlações para identificar se o aumento de produção nacional, resulta em melhoria de qualidade de vida. Por outro lado, compara se o PIB, provocará a diminuição no custo da cesta básica. E ainda compara o aumento de custo da cesta básica provocará a diminuição na qualidade de vida.

Portanto, a pesquisa foi construída com a seguinte problematização: Poderá ocorrer correlação entre o PIB e o IDH, ou ainda entre o PIB e a cesta básica, ou mesmo a correlação entre IDH e a cesta básica? Portanto, o objetivo da pesquisa é verificar as possíveis correlações entre o PIB e o IDH, ou entre o PIB e a cesta básica e entre o IDH e a cesta básica.

\section{REFERENCIAL TEÓRICO}

Na pesquisa buscam-se os resultados obtidos em órgãos ou institutos de controles estatísticos, site responsáveis pelos índices, artigos científicos ou literaturas. Para fundamentar as informações compiladas.

O foco é determinar pelo coeficiente de correlação da teoria de Pearson; se o cálculo gera a comparação entre duas variáveis e indica o grau de correlação. Assim, evidencia-se os aspectos conceituais, por meio da metodologia matemática para obter resultados na pesquisa relevante para conclusão comparativa.

Na Tabela 1 encontram-se as fontes de pesquisa que contribuíram na compilação deste trabalho para orientações e comparações sobre o ponto de vista de outros países, sendo de fundamental importância no desenvolvimento prático e teórico 
ANÁLISE DE CORRELAÇÕES ENTRE INDICADORES ECONÔMICOS: PIB, CESTA BÁSICA E IDH

Tabela 1 - Pesquisa anteriores realizadas sobre o tema

\begin{tabular}{l|l|l|c}
\hline \multicolumn{1}{c|}{ Autor } & \multicolumn{1}{c|}{ Trabalho } & \multicolumn{1}{c|}{ Objetivos } & Ano \\
\hline Bueno, E.P. & $\begin{array}{l}\text { O Índice de Desenvolvimento } \\
\text { Humano (IDH): Avaliação de } \\
\text { seus pressupostos Teóricos e } \\
\text { Metodológicos }\end{array}$ & $\begin{array}{l}\text { Uma avaliação, das características básicas do } \\
\text { Índice IDH }\end{array}$ & 2007 \\
\hline $\begin{array}{l}\text { Domingues, } \\
\text { F. }\end{array}$ & $\begin{array}{l}\text { Além do PIB: novos indicadores } \\
\text { de prosperidade como fonte para } \\
\text { o Jornalismo Econômico }\end{array}$ & $\begin{array}{l}\text { Um acompanhamento maior, por parte do } \\
\text { jornalismo econômico, das variáveis que } \\
\text { influenciam a qualidade de vida }\end{array}$ & 2017 \\
\hline Silva, R. B. & $\begin{array}{l}\text { Uma Análise comparativa do } \\
\text { custo da cesta básica }\end{array}$ & $\begin{array}{l}\text { Trazer informações sobre a evolução dos preços } \\
\text { dos produtos que integram a cesta básica }\end{array}$ & 2018 \\
\hline Souza, A. & $\begin{array}{l}\text { Coeficiente de correlação de } \\
\text { Pearson e coeficiente de } \\
\text { correlação de Spearman }\end{array}$ & $\begin{array}{l}\text { Identificar qual o tipo de relação existente entre a } \\
\text { oferta de etanol e alguns fatores socioeconômicos } \\
\text { do setor }\end{array}$ & 2019 \\
\hline $\begin{array}{l}\text { Stantion, } \\
\text { J.M, Galton } \\
\text { Pearson and } \\
\text { the Peas }\end{array}$ & $\begin{array}{l}\text { A brief history of linear } \\
\text { instructors for }\end{array}$ & $\begin{array}{l}\text { Este artigo apresenta uma breve história de como } \\
\text { Galton originalmente derivou e aplicou a regressão } \\
\text { linear em problemas de hereditariedade }\end{array}$ & 2001 \\
\hline $\begin{array}{l}\text { Yang, C. Ko- } \\
\text { Hsin }\end{array}$ & $\begin{array}{l}\text { Human development and } \\
\text { government effectiveness }\end{array}$ & $\begin{array}{l}\text { Este artigo, no entanto, visa fornecer uma imagem } \\
\text { mais ampla de como o desempenho do governo } \\
\text { pode influenciar no desenvolvimento humano de } \\
\text { um país usando indicadores agregados de } \\
\text { governança para retratar um governo eficaz }\end{array}$ & 2010 \\
\hline
\end{tabular}

Fonte: Elaborado pelos autores.

\section{PIB - PRODUTO INTERNO BRUTO}

Segundo o Instituto Brasileiro de Geografia e Estatística (IBGE,1990), o PIB, indica a soma monetária de todos os bens, gastos públicos e serviços finais; considera-se nesta soma também o resultado da balança comercial do país, ou seja, exportação menos importação. Para evitar dupla contagem, de produções de Países, Estados ou Cidades, contabiliza-se o preço do produto pronto. Pois, as etapas intermediárias, como matéria-prima e serviços; são somadas ao valor final.

Na medição do PIB o IBGE utiliza-se do preço que chega ao consumidor para também considerar os impostos sobre os produtos comercializados. Logo, percebese que o PIB se trata de um indicador que mede a produção final de um período.

Desde 1.990 o IBGE tornou-se a instituição de controle estatístico responsável pela medição do PIB. Mostra-se este por 3 ópticas, ambas devem apresentar o resultado do cálculo no mesmo valor; independente da escolha de visão, sendo estas: a despesa, o rendimento ou a produção: 
ANÁLISE DE CORRELAÇÕES ENTRE INDICADORES ECONÔMICOS: PIB, CESTA BÁSICA E IDH

Óptica da produção composta pela soma de valores da produção de todos os setores da economia, provenientes da indústria, agropecuária e serviços, incluem -se os impostos indiretos e depreciação do capital e exclui os subsídios governamentais. Descrição matemática $\mathrm{PIB}=$ soma do valor acrescido bruto mais impostos sobre produtos e serviços líquidos de subsídios.

Conforme a Tabela 2, que representa o valor acrescentado bruto; percebese o valor total igual ao valor do produto final.

Tabela 2 - Demonstração do valor acrescido

\begin{tabular}{l|l|l}
\multicolumn{1}{c|}{ Fases do processo produtivo } & \multicolumn{1}{|c}{ Produto final (para mil kg) } & \multicolumn{1}{c}{ Valor acrescentado } \\
\hline Extração de trigo & $\mathrm{R} \$ 15,00$ & $\mathrm{R} \$ 15,00$ \\
\hline Produção de farinha & $\mathrm{R} \$ 33,50$ & $\mathrm{R} \$ 18,50$ \\
\hline Produção de pão & $\mathrm{R} \$ 49,00$ & $\mathrm{R} \$ 15,50$ \\
\hline & & Total $=\mathrm{R} \$ 49,00$ \\
\hline
\end{tabular}

Fonte: Dados do IBGE, extraído do site Dicionário Financeiro, 2020. Elaborado pelos autores.

Óptica da renda inclui-se as remunerações dos empregados, impostos indiretos, lucros não distribuídos e depreciação do capital. Os agentes da economia que geram a renda, citam-se a seguir. Agentes geradores na ótica da renda: salários provenientes de rendimentos, aluguéis que provém de arrendamentos, lucros de rendimentos de capitais, juros de aplicações de capitais, rendimentos das amortizações, recursos de arrecadações de impostos indiretos, subtraem-se do somatório os subsídios à produção.

Óptica da despesa composta pela soma dos gastos: nos consumos das famílias e do governo, inclui variações de estoques e a soma das operações exportações e importações.

Para melhor entender o IBGE descreve-se a operação matemática.

Soma-se: a despesa em consumo, a despesa do Estado por meio de gastos públicos, a despesa gerada pelos investimentos das empresas, menos o resultado da balança comercial que são as exportações subtraídas às importações.

No entendimento da autora Yang (2010, p.5), explica-se pela óptica econômica que o governo, por meio de instituições produz de forma positiva o impacto no desenvolvimento humano, quando é eficaz no aumento do PIB. Contudo, a autora afirma 
ANÁLISE DE CORRELAÇÕES ENTRE INDICADORES ECONÔMICOS: PIB, CESTA BÁSICA E IDH

que altas taxas de crescimento do PIB não significa de forma automática em níveis elevados no desenvolvimento humano.

\section{CESTA BÁSICA}

Segundo o Departamento Intersindical de Estática e Estudos Socioeconômicos (DIEESE, 2016, p.1), o levantamento de preços em lista de itens de alimentos considerados essenciais por meio da Pesquisa Nacional de Cesta Básica de Alimentos (PNCBA, 2016) os preços pesquisados para cálculo do Índice de Custo de Vida (ICV).

O (DIEESE, 2016) no ano de 2016 ampliou o perímetro de pesquisa para as 27 capitais brasileiras. Relaciona-se na Tabela 3 a lista das capitais:

Tabela 3 - Relação de capitais com cesta básica

\begin{tabular}{|c|c|c|c|}
\hline Capital & Data de início & Capital & Data de início \\
\hline São Paulo & jan/59 & Goiânia & dez/94 \\
\hline Porto Alegre & jan/77 & Aracajú & set/95 \\
\hline Belo Horizonte & jul/79 & Manaus & out/08 \\
\hline Rio de Janeiro & jan/83 & Campo Grande & nov/12 \\
\hline Salvador & $\mathrm{abr} / 83$ & Cuiabá & $\mathrm{jan} / 16$ \\
\hline Curitiba & jun/83 & Palmas & $\mathrm{jan} / 16$ \\
\hline Florianópolis & mai/85 & Maceió & $\mathrm{jan} / 16$ \\
\hline Brasília & $\mathrm{jan} / 86$ & São Luís & $\mathrm{jan} / 16$ \\
\hline Fortaleza & set/86 & Teresina & $\mathrm{jan} / 16$ \\
\hline Recife & $\mathrm{fev} / 87$ & Macapá & $\mathrm{jan} / 16$ \\
\hline Belém & ago/87 & Rio Branco & jan/16 \\
\hline João Pessoa & jul/88 & Porto Velho & jan/16 \\
\hline Vitória & out/88 & Boa vista & $\mathrm{jan} / 16$ \\
\hline Natal & fev/91 & & \\
\hline
\end{tabular}

Fonte: DIEESE, 2.016. Elaborado pelos autores. 
ANÁLISE DE CORRELAÇÕES ENTRE INDICADORES ECONÔMICOS: PIB, CESTA BÁSICA E IDH

O (DIEESE, 2016), usa em suas pesquisas as listas cadastradas nas respectivas capitais, listadas na tabela 2; vale-se das fontes como associações e sindicatos, pois os estabelecimentos têm seus dados registrados nestas instituições e quando não houver usase o método de pesquisa eletrônica ou in loco.

A responsabilidade pelo cálculo do indicador de cesta básica é do DIEESE (2016)), pois em janeiro de 2.016 atualizou a metodologia da pesquisa. Este, escolheu 13 alimentos para atender em um mês, o sustento de um trabalhador com faixa etária adulta. Define-se por região para diferenciar os bens e quantidades, devido os hábitos alimentares diferenciados.

Verifica-se a seguir na Tabela 4, as quantidades mensais, estipuladas na composição da cesta básica, abrange-se por região no território nacional.

Tabela 4 - Provisões mínimas por regiões da Cesta Básica

\begin{tabular}{c|c|c|c|c}
\hline Alimentos & Região 1 & Região 2 & Região 3 & Nacional \\
\hline Carne & $6,0 \mathrm{~kg}$ & $4,5 \mathrm{~kg}$ & $6,6 \mathrm{~kg}$ & $6,0 \mathrm{~kg}$ \\
\hline Leite & 7,51 & 6,01 & 7,51 & 15,01 \\
\hline Feijão & $4,5 \mathrm{~kg}$ & $4,5 \mathrm{~kg}$ & $4,5 \mathrm{~kg}$ & $4,5 \mathrm{~kg}$ \\
\hline Arroz & $3,0 \mathrm{~kg}$ & $3,6 \mathrm{~kg}$ & $3,0 \mathrm{~kg}$ & $3,0 \mathrm{~kg}$ \\
\hline Farinha & $1,5 \mathrm{~kg}$ & $3,0 \mathrm{~kg}$ & $1,5 \mathrm{~kg}$ & $1,5 \mathrm{~kg}$ \\
\hline Batata & $6,0 \mathrm{~kg}$ & - & $6,0 \mathrm{~kg}$ & $6,0 \mathrm{~kg}$ \\
\hline $\begin{array}{c}\text { Legumes } \\
\text { (Tomate) }\end{array}$ & $9,0 \mathrm{~kg}$ & $12,0 \mathrm{~kg}$ & $9,0 \mathrm{~kg}$ & $9,0 \mathrm{~kg}$ \\
\hline Pão francês & $6,0 \mathrm{~kg}$ & $6,0 \mathrm{~kg}$ & $6,0 \mathrm{~kg}$ & $6,0 \mathrm{~kg}$ \\
\hline Café em pó & $600 \mathrm{gr}$ & $300 \mathrm{gr}$ & $600 \mathrm{gr}$ & $600 \mathrm{gr}$ \\
\hline $\begin{array}{c}\text { Frutas (Banana) } \\
90 \mathrm{unid}\end{array}$ & $90 \mathrm{unid}$ & $90 \mathrm{unid}$ & $90 \mathrm{unid}$ \\
\hline Açúcar & $3,0 \mathrm{~kg}$ & $3,0 \mathrm{~kg}$ & $3,0 \mathrm{~kg}$ & $3,0 \mathrm{~kg}$ \\
\hline Banha/Óleo & $750 \mathrm{gr}$ & $750 \mathrm{gr}$ & $900 \mathrm{gr}$ & $1,5 \mathrm{~kg}$ \\
\hline Manteiga & $750 \mathrm{gr}$ & $750 \mathrm{gr}$ & $750 \mathrm{gr}$ & $900 \mathrm{gr}$ \\
\hline
\end{tabular}

Fonte: Decreto Lei número 399 de 1938- Conversão de quantidades diárias para mensais. Elaborado pelos autores.

Conforme o DIEESE (2016, p.2), observa-se a seguir as descrições por regiões: 


\section{ANÁLISE DE CORRELAÇÕES ENTRE INDICADORES ECONÔMICOS: PIB, CESTA BÁSICA E IDH}

Região 1 - Estados do Espírito Santo, Minas Gerais, Rio de Janeiro, São Paulo, Goiás e Distrito Federal.

Região 2 - Estados de Alagoas, Bahia, Ceará, Maranhão, Paraíba, Pernambuco, Piauí, Rio Grande do Norte, Sergipe, Acre, Amapá, Amazonas, Pará, Rondônia, Roraima e Tocantins.

Região 3 - Estados do Paraná, Rio Grande do Sul, Santa Catarina, Mato Grosso e Mato Grosso do Sul.

Nacional - Cesta normal média para a massa trabalhadora em atividades diversas e para todo o território nacional.

Os locais da coleta definem-se, por meio da POF Pesquisa de Orçamentos Familiar de 2008/2009 do IBGE. Considera-se os gastos médios mensais pelas famílias de 1 a 3 salários mínimos e os locais de aquisições dos itens da cesta básica.

O IBGE divulga os dados da POF a nível Brasil, grandes regiões e por situação urbana ou rural. A pesquisa dos orçamentos familiares define-se, por amostra mestra, os setores são selecionados dentre aqueles que compõem a referida amostra.

Variados tipos de locais são identificados pela POF, dividem-se em 4 principais tipos de comércios, sendo:

(1) - Supermercado: supermercado, hipermercado, mercearia, armazém, empório e outros;

(2) - Feira: feira-livre, mercado municipal, hortifruti, sacolão, quitanda, frutaria, fruteiro, verdureira, feira de frutas e outros;

(3) - Açougue: açougue e casa de carne; e,

(4) - Padaria: padaria, confeitaria, casa de pães, casa de doce, panificadora, posto de pão, depósito de pão e outros.

Conforme a metodologia do DIEESE na cesta básica nacional de alimentos os preços são pesquisados nos estabelecimentos em cada região no Brasil. Apuração de preço mensal para a Cesta Básica e conforme segue.

O preço médio de cada produto, multiplicado pelas quantidades definidas no Decreto Lei $\mathrm{n}^{\circ} 399$, indica o gasto mensal do trabalhador com cada produto, cuja soma é o custo mensal da Cesta Básica. Obtido o valor da cesta, é feito o cálculo das horas que o trabalhador que ganha salário mínimo precisa trabalhar para comprar a Cesta Básica de Alimentos. DIEESE (2.016, p.3)

Conforme o DIEESE (2016), por sua metodologia o cálculo do valor mensal da Cesta Básica nacional; se obtém na Constituição de outubro de 1988. Onde determina-se pelo número de 220 horas, indicado na sequência da fórmula:

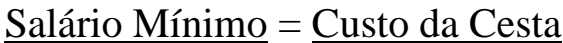


ANÁLISE DE CORRELAÇÕES ENTRE INDICADORES ECONÔMICOS: PIB, CESTA BÁSICA E IDH

220 $\mathrm{X}$

$\mathrm{X}=\underline{\text { Custo da Cesta }} \quad \mathbf{x} 220$

Salário Mínimo

No cálculo o DIEESE, obtém o custo em diferentes capitais para manter a alimentação básica de um mês de trabalho de um trabalhador.

Segundo Silva, 2018, o DIEESE acompanha mensalmente o custo da Cesta Básica, pois desde janeiro de 1959, o Dieese passou a acompanhar mensalmente o custo da Cesta Básica Nacional, realizando a pesquisa da Cesta Básica Nacional em capitais do país e acompanhando mensalmente a evolução de preços dos treze produtos que compõe a cesta, assim como o gasto mensal que um trabalhador teria para adquiri-la.

\section{IDH - ÍNDICE DE DESENVOLVIMENTO HUMANO}

No Relatório de Desenvolvimento Humano publicado em 2014 pelo Programa das Nações Unidas para o Desenvolvimento (PNUD, 1990), informa-se que o controle de divulgação do IDH é da PNUD desde 2010. E ainda este PNUD tem ligação na Organização das Nações Unidas (ONU).

O Índice de Desenvolvimento Humano Municipal (IDHM), segundo o Atlas Brasil, (2013), informa que em 1998 o Brasil, apresentou-se como uns dos pioneiros na adaptação do IDH com a abrangência municipal. A Gâmbia nesta época tornou-se exemplo, pelas criações de diferentes variáveis incorporadas à taxa de alfabetização adulta e à expectativa de vida escolar.

Em 2013, segundo o PNUD (2020), O Instituto de Pesquisa Econômica Aplicada (IPEA) e a Fundação João Pinheiro realizam o site para consulta eletrônica do IDH por regiões municipais brasileiras, nas bases dos dados levantados pelos Censos feitos pelo IBGE de 1.991, 2.000 e 2.010.

O IDH tornou-se um índice de referência mundial e chave para os objetivos das Nações Unidas. Sintetiza-se a compreensão e fomenta-se o debate de temas; não completados pelo IDH como: democracia, participação, equidade, sustentabilidade, felicidade da pessoa ou o melhor lugar do mundo para viver. 


\section{ANÁLISE DE CORRELAÇÕES ENTRE INDICADORES ECONÔMICOS: PIB, CESTA BÁSICA E IDH}

Segundo a PNUD (2020), desde 1990 no Brasil; se mede o IDH pelo progresso de uma nação e mensura-se pela análise de 3 pontos educação, renda e saúde. A seguir melhor se descrevem os pontos:

Educação o acesso ao conhecimento mede-se para adulto e criança, sendo: a média de anos de educação de adulta recebida a partir dos 25 anos; se verifica na fase infantil as taxas de matrículas, analisam-se o tempo, se mantém os padrões de períodos e a expectativa durante a vida na infância para o início na vida escolar,

renda esta mede-se pelo padrão de vida por meio da RNB Renda Nacional Bruta per capita, usa-se o dólar como moeda e 2005 como ano base para expressar o PPP Poder de Paridade de Compra, saúde mede-se pela expectativa de vida, a longevidade saudável.

Informa ainda (PNUD, 1990) que se criou o IDH como um contraponto do PIB e por meio do IDH mede-se a média indicada nas conquistas de desenvolvimento humano básico em um país. Usa-se no Brasil pelo governo Federal e pela administração Municipal o IDH-M Índice de Desenvolvimento Humano Municipal. O IDH-M consiste em ajuste metodológico publicado em 1998 ao IDH Global.

\section{HIPÓTESES}

As avaliações de dados históricos entre as variáveis, se testam pelos resultados apresentados, por meio dos cálculos submetidos. Para verificações de correlações entre as variáveis. Portanto, foram definidas as seguintes hipóteses:

$\mathrm{H}_{1}$. Existe correlação entre as variáveis PIB em relação a Cesta Básica

$\mathrm{H}_{2}$. Existe correlação entre as variáveis PIB em relação ao IDH

$\mathrm{H}_{3}$. Existe correlação entre as variáveis Cesta Básica e IDH

Ao verificar as hipóteses, se buscará determinar os resultados para verificar o grau de significância entre os indicadores, que servirão de reflexos em decisões pertinentes a determinado sistema econômico. 
ANÁLISE DE CORRELAÇÕES ENTRE INDICADORES ECONÔMICOS: PIB, CESTA BÁSICA E IDH

\section{METODOLOGIA}

O estudo proposto de forma comparada e descritiva, obtém-se por fontes destas disponíveis em websites pelas instituições de controles estatísticos. O IBGE disponibiliza o valor do PIB no trimestre e ao ano, o controle de preço da Cesta Básica realiza-se mensal pelo Dieese e o IDH do Brasil tem a base de correção no censo municipal de dez em dez anos; porém este índice possui divulgação a anual pela PNUD.

Apurou-se nesta pesquisa os resultados no período de 2.007 a 2.018. As variáveis assumem os valores anuais; para verificações comparativas das relações entre os indicadores econômicos e usa-se a teoria de Pearson.

"O coeficiente de correlação de Pearson é uma medida do grau de relação linear entre duas variáveis quantitativas x e y" (Stanton, 2001, p. 9).

Segundo Santos e Pinto (2017, p.17-18), confirma-se a importância da definição de correlação entre as variáveis para entendê-las.

\footnotetext{
No estudo do relacionamento entre duas variáveis, a definição de correlação é de suma importância para o entendimento das variações existentes. A análise de correlação avalia o grau de relacionamento linear entre duas variáveis. De acordo com Triola (1999), ao se considerar duas variáveis, o Coeficiente de Correlação Linear amostral $r$ mede o grau de relacionamento linear entre os dados emparelhados de $\mathrm{x}$ e y em uma amostra, e o seu valor pode ser calculado pela equação 1.De acordo com Triola (1999) o Coeficiente de Correlação Linear amostral $r$ deve estar sempre compreendido entre -1 e +1 , sendo que valores próximos de -1 e +1 demonstram maior correlação e valores próximos de zero pode-se concluir pela ausência de correlação entre as duas variáveis $\mathrm{x}$ e $\mathrm{y}$ analisadas.
}

Denomina-se a equação de Pearson pela fórmula:

$$
\rho=\frac{\sum_{i=1}^{n}\left(X_{i}-\underline{X}\right)\left(Y_{i}-\underline{Y}\right)}{n \sigma_{x} \sigma_{y}} \quad \text { (1) equação de Pearson }
$$

Segundo as variáveis X e Y Pearson descreve-se, abaixo onde: 
ANÁLISE DE CORRELAÇÕES ENTRE INDICADORES ECONÔMICOS: PIB, CESTA BÁSICA E IDH

Xi é a i - ésima observação da Variável $\mathrm{X}, \mathrm{i}=1,2,3,4, \ldots, \mathrm{k}, \ldots, \mathrm{n}$ Yi é a $\mathrm{i}$ - ésima observação da Variável $Y, i=1,2,3,4, \ldots, k, \ldots, n$ Oxx é o desvio padrão da variável X Ơy é o desvio padrão da variável Y. Sendo o "n” para ambas as variáveis X e Y.

Evidencia-se que ambas deverão necessariamente ter o mesmo tamanho n. Sempre que o coeficiente de Pearson determina, como um número real compreendido entre -1 e 1 .

Quanto mais próximo o resultado da variável for de -1 ou de 1. mais forte será apresentada a correlação entre as variáveis de estudo. A diferença é que a correlação próxima de 1 considera-se forte e positiva, analogamente, a correlação próxima de -1 considera-se forte negativa. Correlações intermediárias a estes valores perdem a sua força à medida que o valor da correlação se aproxima de zero.

O coeficiente de Pearson, ou simplesmente correlação, mede exatamente este grau de associação linear entre duas variáveis em pesquisa.

\section{RESULTADOS E DISCUSSÕES}

Observa-se na Tabela 5 o painel de resultados para os índices econômicos, medidos de forma anual, sendo que para os valores dos indicadores PIB e Cesta Básica expressam-se em valores monetários e o último representado pelo IDH compreende a faixa de 0 a 1.

Tabela 5 - Painel de Resultado dos índices Econômicos

\begin{tabular}{c|c|c|c}
\hline ANO & PIB-A - R\$ & CESTA BÁSICA - R\$ & IDH-C \\
\hline 2007 & 2597,61 & 166,84 & 0,80 \\
\hline 2008 & 2889,72 & 205,88 & 0,807 \\
\hline 2009 & 3143,02 & 206,94 & 0,813 \\
\hline 2010 & 3674,96 & 217,28 & 0,699 \\
\hline 2011 & 4143,01 & 235,52 & 0,718 \\
\hline
\end{tabular}


ANÁLISE DE CORRELAÇÕES ENTRE INDICADORES ECONÔMICOS: PIB, CESTA BÁSICA E IDH

\begin{tabular}{c|c|c|c}
\hline 2012 & 4392,09 & 256,04 & 0,742 \\
\hline 2013 & 4844,82 & 290,22 & 0,754 \\
\hline 2014 & 5521,26 & 302,69 & 0,757 \\
\hline 2015 & 5904,33 & 340,90 & 0,758 \\
\hline 2016 & 6259,23 & 399,49 & 0,758 \\
\hline 2017 & 6559,94 & 381,99 & 0,759 \\
\hline 2018 & 6645,22 & 383,48 & 0,759 \\
\hline
\end{tabular}

Fonte: Dados da Pesquisa.

A técnica de estatísticas, por meio de resultados apurados e descritos no quadro 5, são os valores atribuídos para informar os resultados de cada indicador, onde A representa variável denominada PIB, B representa a Cesta Básica e C indica o resultado do IDH.

Definidas as hipóteses, a nula e a alternativa entre as variáveis de estudo.

Representa-se as hipóteses:

$\mathrm{H}: \rho=0$ não existe relacionamento linear,

H: $\rho \neq 0$ existência de relacionamento linear.

Na Tabela 6 se descrevem os resultados dos cálculos de correlações, o intervalo, o grau de liberdade $(\mathrm{gl})$, bem como o nível de significância $(\alpha)$ e o percentual, total apurado em cada variável dos índices, o fator da variável e os resultados das hipóteses. 
ANÁLISE DE CORRELAÇÕES ENTRE INDICADORES ECONÔMICOS: PIB, CESTA BÁSICA E IDH

Tabela 6 - Descrição cálculos de índices econômicos por meio de hipóteses

\begin{tabular}{|c|c|c|c|c|c|c|c|}
\hline Teste $\mathrm{t}$ & correlação ( & $\mathbf{N}$ & gl & $\alpha$ & $\mathbf{T}$ & p-valor & resultado \\
\hline pib-cesta & 0,980413758 & 12 & 10 & $5 \%$ & 15,74185388 & 0,00000002 & $\begin{array}{c}\text { Existe } \\
\text { relacionamento } \\
\text { linear forte (1) }\end{array}$ \\
\hline pib-idh & $-0,320083566$ & 12 & 10 & $5 \%$ & $-1,068402498$ & 0,31045104 & $\begin{array}{c}\text { Sem } \\
\text { relacionamento (2) }\end{array}$ \\
\hline cesta-idh & $-0,218959585$ & 12 & 10 & $5 \%$ & $-0,709630958$ & 0,49414688 & $\begin{array}{c}\text { Sem } \\
\text { relacionamento (3) }\end{array}$ \\
\hline
\end{tabular}

ElaboFonte: Dados da Pesquisa, junho de 2020.

$$
\rho=0,98 \text { na correlação PIB x Cesta Básica (1) }
$$

A correlação (1) sugere uma ascensão linear indica-se uma associação linear forte entre as variáveis.

Por outro lado, não há evidências de associação entre as variáveis PIB - IDH (2) e também não encontradas evidências de associações entre cesta básica e IDH (3).

Argumenta Bueno (2007, p.54), em relação ao IDH para expor a tentativa na criação de outro indicador para correção de falhas no PIB per capita na medição de seu desenvolvimento. Este ainda menciona os exemplos de outros autores.

Nesta revisão da literatura, inicialmente destacamos a análise elaborado por Mancero (2001, p. 20) que, em relação ao IDH, questionou o seguinte: "Vale destacar que éste no es el primer intento por construir um indicador que corrija los defectos del PIB "per capita” em la medición del desarrolo". Neste sentido, cita trabalho realizado por McGillivray (1991) que aponta exemplos de estudos alternativos, como o índice de "padrão de vida" elaborado por Drewnowski e Scott (1966); o "índice de desenvolvimento" de McGranahan et al. (1972) e o "índice de qualidade de vida física" (PQLI) organizado por Morris (1979). De acordo com o autor mencionado, o uso destes índices não prosperou, entre outras razões, por sua "redundância" com respeito ao PIB "per capita", pois todos eles apresentavam uma correlação alta com a renda. Neste sentido, eles não constituíam, realmente, uma contribuição prática como medida do bemestar e nem uma verdadeira inovação. 
ANÁLISE DE CORRELAÇÕES ENTRE INDICADORES ECONÔMICOS: PIB, CESTA BÁSICA E IDH

\section{CONSIDERAÇÕES FINAIS}

Neste artigo se apresentam os resultados, por meio do coeficiente Pearson, na pesquisa obtém-se o período de forma anual para todos os indicadores econômicos. Propôs-se a análise comparativa para verificar as correlações entre as variáveis atribuídas aos valores dos índices respectivos ao PIB, Cesta Básica e IDH.

$\mathrm{Na}$ teoria de Pearson as comparações usam duas variáveis, por vez, formam se as hipóteses neste critério para apurar o grau de influência comparativa. Os objetivos de testes das hipóteses, buscam os resultados para possibilitar as análises.

Entre o PIB e a Cesta Básica a correlação determina-se pelo coeficiente de Pearson o valor de 0,98 . Pearson atribui que o valor de 1 , que sugere uma ascensão linear e indica uma associação linear forte entre as variáveis. Portanto indica-se forte correlação para o PIB em relação a Cesta Básica representado resultado 0,980413758, a um nível de significância $\alpha=5 \%$

Por outro lado, o resultado de $-0,320083566$ não representa a evidência de associação entre as variáveis PIB em relação ao IDH. E o resultado de -0,218959585 indica que não tem evidências de associação também entre as variáveis cesta básica e IDH, sempre mencionado a um nível de significância de $\alpha=5 \%$

Contudo, a pesquisa poderá auxiliar em futuras análises para atualizações, acompanhamentos de desempenhos, verificar grau de influência comparativa; sejam para as variáveis expostas na pesquisa ou novas. A pesquisa contribui na elaboração de políticas públicaso, pois, é nítido que o fato do país aumentar a sua produção não impacta em melhor desenvolvimento e qualidade de vida para população, logo, maior produção amplia as diferenças, aumentando a concentração. Este fato inidica a necessidade de revisão da legislação brasileira, em especial à questões tributárias e distributivas de renda e produção.

A pesquisa limita-se em definir as comparações, porém poderá servir de modelo para análises similares. 
ANÁLISE DE CORRELAÇÕES ENTRE INDICADORES ECONÔMICOS: PIB, CESTA BÁSICA E IDH

\section{REFERÊNCIAS}

Bueno, Edir de Paiva. (2007). O Índice de Desenvolvimento Humano (IDH): avaliação de seus pressupostos teóricos e metodológicos. Boletim Goiano de Geografia vol. 27 n.3. 2007. Universidade Federal de Goiás. jul. - dez. Goiás. Disponível em: <https://revistas.ufg.br/bgg/article/view/6020/9875> Acessado 15 jun. 2020.

Dias, Elisângela, Gonçalves, Juliana, Marques, Rui e Pereira, Leonardo. (2020). Produto Interno Bruto: o que é e como é calculado o PIB. Dicionário Financeiro 7 Graus Lda. Portugal. Disponível em: 〈https://www.dicionariofinanceiro.com/pib/> Acessado 23 jun. 2020.

DIEESE - Departamento Intersindical de Estatística e Estudo Socioeconômicos. (2016). Metodologia da Pesquisa Nacional da Cesta Básica de Alimentos. 15 fev. Centro - São Paulo. Disponível em:

$\langle$ https://www.dieese.org.br/metodologia/metodologiaCestaBasica2016.pdf $>$ Acessado 15 jun. 2020.

Domingues, Filipe. (2017). Além do PIB: novos indicadores de prosperidade como fonte para o jornalismo econômico. Revista Communicare. vol.17. n.1 primeiro semestre. São Paulo. Disponível em: <https://casperlibero.edu.br/wpcontent/uploads/2017/06/Artigo-3.pdf > Acessado 15 set. 2020.

FIA - Fundação Instituto de Administração. (2019). PIB (Produto Interno Bruto): guia completo do PIB de A a Z. 02 de mar. São Paulo. Disponível em:

<https://fia.com.br/blog/pib/> Acessado 15 jun. 2020.

IBGE - Instituto Brasileiro de Geográfia e Estatística. Cálculo do PIB. (2015). Rio de Janeiro. Disponível em: 〈https://www.ibge.gov.br/explica/pib.php〉 Acessado 15 jun. 2020.

PNUD - Programa das Nações Unidas para o Desenvolvimento. (2020). Objetivos do Desenvolvimento Sustentável: o que é IDH. Brasília. Disponível em: <https://www.br.undp.org/content/brazil/pt/home/idh0/conceitos/o-que-e-o-idh.html > Acessado 15 jun. 2020.

PNUD - Programa das Nações Unidas para o Desenvolvimento (2020). Relatório do Desenvolvimento Humano. Brasília. Disponível em:

$<$ https://www.undp.org/content/dam/brazil/docs/RelatoriosDesenvolvimento/undp-brhdr_portugues-2014.pdf $>$ Acessado 26 set. 2020.

Santos, Anna Carolina Silva e Pinto, Rafael Lucas Machado. (2017). Aplicação da análise de correlação e regressão linear simples no setor sucroenergético brasileiro. Exacta vol.16 n.2. 2018. Universidade Nove de Julho. 26 de set. São Paulo. Disponível em: <https://www.redalyc.org/jatsRepo/810/81058960010/html/index.html > Acessado 23 jun. 2020.

Silva, Renata Boscolo da. (2018). Uma Análise comparativa do custo da cesta básica. Trabalho de Conclusão de Curso (Bacharelado em Ciências Econômicas). Faculdade de Administração, Ciências Contábeis e Economia, Universidade Federal da Grande Dourados. Dourados. Mato Grosso do Sul. Disponível em: 


\section{ANÁLISE DE CORRELAÇÕES ENTRE INDICADORES ECONÔMICOS: PIB, CESTA BÁSICA E IDH}

<http://repositorio.ufgd.edu.br/jspui/bitstream/prefix/2930/1/RenataBoscolodaSilva.pdf $>$ Acessado em : 21 set. 2020.

Sousa, Áurea. (2019). Coeficiente de correlação de Pearson e coeficiente de correlação de Spearman: o que medem e em que situações devem ser utilizados?. Correio dos Açores: Matemática. 21 de mar. de 2019. P.19. Universidade dos Açores. 21 de mar. Portugal. Disponível em: 〈http://hdl.handle.net/10400.3/5365〉 Acessado 15 set. 2020.

Stantion, Jeffrey (2001). M. Galton Pearson and the Peas: a brief history of linear regression for statistics instructors. Journal of Statistical Education, 9, 3. New York. Disponível em: 〈http://jse.amstat.org/v9n3/stanton.html> Acessado 15 jun. 2020.

Yang, Christina Ko-Hsin. (2010). Human development and government effectiveness. Dissertação Master of Public Policy - Georgetown Public Policy Institute, Faculty of the Graduate School of Arts and Sciences, Georgetown University, Georgetown. Apr. 13, Washington. Disponível em:

$<$ http://citeseerx.ist.psu.edu/viewdoc/download?doi=10.1.1.1029.6390\&rep=rep1\&type $=$ pdf $>$ Acessado em 15 jun. 2020. 